\title{
FROM THE NEUTRAL THEORY TO A COMPREHENSIVE AND MULTISCALE THEORY OF ECOLOGICAL EQUIVALENCE
}

\author{
FrançOIS MUNOZ \\ Université de Montpellier, UMR-AMAP, TA A-51/PS2 \\ 34398 Montpellier cedex 5, France \\ French Institute of Pondicherry \\ Pondicherry 605001, India \\ E-MAIL: FRANCOIS.MUNOZ@CIRAD.FR \\ Philippe Huneman \\ Université Paris I Sorbonne, IHPST \\ 75006 Paris, France \\ E-MAIL: PHILIPPE.HUNEMAN@GMAIL.COM
}

KEYWORDS

neutral theory, community ecology, ecological equivalence, causal explanation, null hypothesis, philosophy of ecology

\section{ABSTRACT}

The neutral theory of biodiversity assumes that coexisting organisms are equally able to survive, reproduce, and disperse (ecological equivalence), but predicts that stochastic fluctuations of these abilities drive diversity dynamics. It predicts remarkably well many biodiversity patterns, although substantial evidence for the role of niche variation across organisms seems contradictory. Here, we discuss this apparent paradox by exploring the meaning and implications of ecological equivalence.

We address the question whether neutral theory provides an explanation for biodiversity patterns and acknowledges causal processes. We underline that ecological equivalence, although central to neutral theory, can emerge at local and regional scales from niche-based processes through equalizing and stabilizing mechanisms. Such emerging equivalence corresponds to a weak conception of neutral theory, as opposed to the assumption of strict equivalence at the individual level in strong conception. We show that this duality is related to diverging views on hypothesis testing and modeling in ecology. In addition, the stochastic dynamics exposed in neutral theory are pervasive in ecological systems and, rather than a null hypothesis, ecological equivalence is best understood as a parsimonious baseline to address biodiversity dynamics at multiple scales.

The Quarterly Review of Biology, September 2016, Vol. 91, No. 3

Copyright $(2016$ by The University of Chicago Press. All rights reserved.

0033-5770/2016/9103-0006\$15.00 
INTRODUCTION

S INCE the publication of The Unified Neutral Theory of Biodiversity and Biogeography (Hubbell 2001), neutral theory has been very influential but also very controversial in ecology. Across the numerous models that have been elaborated, neutral theory focuses on stochastic variations in individual birth and death rates to predict long-term trends in the composition of communities. Furthermore, the basic concepts of this theory are closely related to the neutral theory of molecular evolution, which has also been influential and controversial among evolutionary biologists from the 1960s (Kimura 1983). At the heart of both approaches is the fundamental equivalence assumption that biological variation among organisms does not reflect any difference in their ability to survive, reproduce, and disperse (Bell 2001). Species coexistence is then a dynamical equilibrium driven by the stochastic variations in speciation and extinction at the regional scale, and in basic life, death, and dispersal dynamics at the local scale (called "neutral dynamics"). The equivalence assumption is traditionally opposed to an exclusive explanatory role of niche variation and competitive exclusion in ecology (Whitfield 2002), and to the correlated idea of natural selection in evolution (Mikkelson 2005).

The neutral theory has shown a remarkably good heuristic value to predict patterns of species abundance distributions (SAD) in a number of ecosystems (Hubbell 1997, 2001; Bell 2001; Chave 2004). Encouraged by this apparent robustness, many studies have applied neutral models to analyze community dynamics in a variety of ecosystems (Volkov et al. 2003; Latimer et al. 2005; Chave et al. 2006). At the same time, variation in survival and reproductive abilities is often observed in real communities and apparently violates the equivalence assumption (Purves and Turnbull 2010). The fact that the theory works well in terms of resulting species-abundance patterns despite the violation of its basic assumption is an apparent paradox (Gewin 2006). A decade and a half after the work of Hubbell (2001), debates and disagreements persist on whether neutral theory provides an explanation for observed species diversity patterns, and on how to test its expectations. Our primary objective here is to clarify the explanatory nature of the theory.

The basic idea of neutral theory is that numerous and repeated "microscopic" individual stochastic variations result, over space and time, in specific patterns of species relative abundances. Central to the debate on neutral theory is the divergence between "weak" and "strong" interpretations of the theory (Bell 2001). The "strong" interpretation considers that neutral theory is refuted if strict fitness equivalence is not met at the individual level. Conversely, in the "weak" interpretation, variation in individual fitness can be counterbalanced and neutral patterns of biodiversity can emerge without strict individual equivalence. In this case, the fact that an observed pattern does not reflect the influence of biological differences does not mean that individual dynamics are actually neutral, but it indicates that the observed pattern does not convey a signature of nonneutral processes. In this regard, the strong interpretation emphasizes basic neutral dynamics at the individual level, while the weak interpretation emphasizes the emergence of neutral patterns.

Here, we analyze the mechanistic understanding of neutral dynamics in the dual interpretations of neutral theory, and show that the issue more broadly relates to what explanation, causality, and hypothesis testing mean in community ecology. In this regard, even a decade and a half after Hubbell's book, some basic questions are still hard to answer: Does neutral theory provide an explanation for real community dynamics? If so, is this explanation causal? Is the neutral theory a null hypothesis against which to analyze observed patterns? Are there instead signatures of both neutral and nonneutral processes in the composition of ecological communities? The nature of explanation is clearly a central issue, since answers to the above questions may rely on what we mean by "signatures" (from a pattern-oriented point of view) and "causal explanation" (from a process-oriented point of view). A basic aim of this paper is then 
to delineate more clearly the nature of ecological equivalence, central to the neutral theory, so as to better assess the status of causation within the theory. We will show that the weak and strong interpretations of neutral theory imply different conceptions of ecological equivalence, which entail a profound epistemic divide. We will emphasize that ecological equivalence can be found at multiple scales under the influence of both neutral and nonneutral dynamics and, therefore, that ecological equivalence can be part of a comprehensive theory of biodiversity dynamics.

This paper will first address the nature of neutral dynamics, and whether the neutral theory can provide a mechanistic framework for biodiversity dynamics and species coexistence. We will especially focus on the relationship between neutral processes and neutral patterns. The second step will question the nature and status of ecological equivalence in terms of equalizing and stabilizing mechanisms, and how it should be used to investigate biodiversity dynamics from local communities to a regional set of species. We will discuss the philosophical nature of the dualism in neutral and nonneutral views, and highlight that spatial and temporal scales are critical aspects of the link between emerging patterns of ecological equivalence and possibly nonneutral underlying processes. We will then distinguish several levels at which mechanisms can be appealed to when one analyzes biodiversity patterns, and reconsider the notion of null hypothesis against which to test the role of niche differences. Actually, a major gap exists currently in ecology between hypotheticodeductive approaches where neutral "random" models represent null hypotheses that should be falsified in favor of alternative models representing the contribution of niche differences and integrative approaches that recognize a genuine role for neutral dynamics and intend to characterize their contribution to community dynamics, possibly in combination with other niche-based processes. The fourth section will distinguish these perspectives and show that they pertain to different conceptions of hypothesis testing. From this analysis, we will show that the traditional dichotomy of neutral and niche-based biodiversity dynamics should be abandoned, and be replaced by a more comprehensive theory of ecological equivalence. When analyzing biodiversity dynamics in terms of ecological equivalence, the focus is no longer on the immediate and local effects of biological differences, but rather on the level and scale at which these differences matter to explain biodiversity patterns. Figure 1 summarizes the overall logic and organization of the paper. Table 1 provides a glossary of the basic concepts of this manuscript.

\section{AN Explanatory THEORY OF \\ UNSPECIFIED INDIVIDUAL INTERACTIONS}

To understand the role of the neutral theory in ecology, one should first focus on the kind of explanation it provides for biodiversity. In neutral communities, species abundances change with time according to stochastic birth, death, and dispersal events, plus speciation events in a large-scale biogeographical metacommunity (Hubbell 2001). This framework is analogous to the neutral theory of population genetics, where species abundance is replaced by allele frequency and speciation by mutation (Kimura 1983). In finite communities, stochastic fluctuations of species abundances (so-called ecological drift, analogous to random genetic drift in population genetics) lead to random extinctions. Without any influx of migrants, a local community will undergo species loss until only one species survives (fixation). Likewise, at the regional scale, the metacommunity will lose species until fixation, unless new species are created by speciation. Although the stochastic variation in speciation, migration, and local birth-death dynamics yields an unpredictable community composition at any given time, statistical patterns such as species abundance distributions can be predicted at equilibrium depending on the balance between speciation, migration, and drift. In the model of Hubbell (2001) with point speciation in the metacommunity, the regional balance of speciation and drift leads to an equilibrium mean number of species (Ewens 1972) and to a log-series 


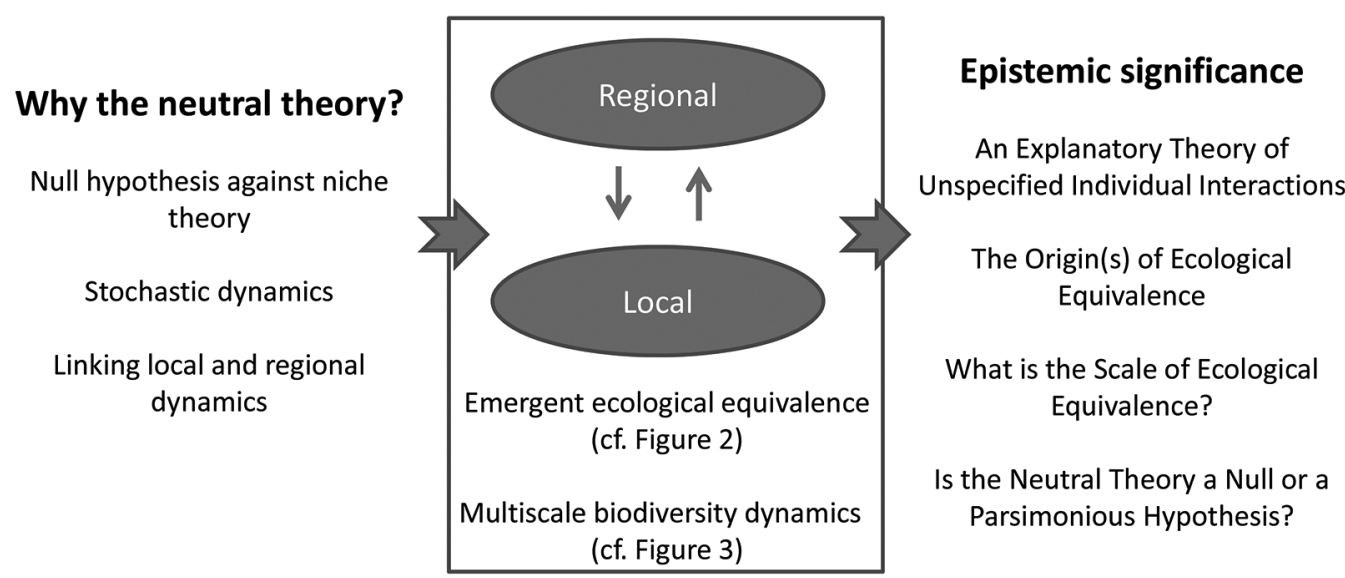

Figure 1. Conceptual Diagram of the Present Paper, with Emphasis on the Central Ecological Equivalence Assumption

The left part shows the basic motivations of the neutral theory, which translates in the middle part into a framework of ecological equivalence and biodiversity dynamics at local and regional scales. The right part introduces the main epistemic aspects that are discussed in the paper.

species abundance distribution. In addition, the relative abundances in a local community depend on the balance of local drift and immigration from this metacommunity. Alternative models have considered different speciation and migration processes, and thus predicted varying biodiversity patterns (e.g., Chave and Leigh 2002; Rosindell et al. 2010). Apart from these variations, any neutral model assumes that there is no influence of biological differences between species on individual dynamics.

Conversely, a niche-based theory of species coexistence (in short, "niche theory") claims that species can coexist or not depending on their niche properties and on specific ecological interactions such as competition or mutualism. Therefore, whereas niche theory is a theory of specified interactions and determinate processes, neutral theory is a theory of unspecified interactions and stochastic dynamics. In the context of population genetics, natural selection is analogous to niche-based processes as a theory of specified interactions between individuals having distinct genotypes. Even though neutral dynamics yield specific species abun- dance distributions, it may still be misleading to refer to them as neutral processes, in the sense that a process is classically defined as a determinate (i.e., specific) cause of some outcomes, which is often characterized in terms of the typicality of some interactions (Ellis 1999; Dowe 2009).

Given that a central motivation of neutral models has been to suggest new explanations of biodiversity patterns, let us now consider the explanatory difference between competing neutral and niche theories. Within the philosophical literature on explanation, explaining can be seen as either providing unifying schemes (Friedman 1974; Kitcher 1976) or characterizing causal relations (Salmon 1984). These views are not necessarily exclusive (e.g., Strevens 2004 for a conciliation), but in ecology a primary objective is to find "mechanistic explanations" to observed patterns, which is a causalist conception of explanation (McGill and Nekola 2010). This conception is indeed central to niche-based coexistence theory, where mechanisms of resource use and interactions at the individual level, depending on niche differences, determine whether species can coexist or 
TABLE 1

Glossary of key concepts

\begin{tabular}{|c|c|}
\hline Term & Definition \\
\hline Neutral biodiversity pattern & $\begin{array}{l}\text { A biodiversity pattern classically summarizes variation in species number and relative } \\
\text { abundances, within and between communities. A neutral biodiversity pattern represents } \\
\text { an equilibrium state resulting from neutral dynamics. }\end{array}$ \\
\hline Neutral dynamics & $\begin{array}{l}\text { A combination of immigration, speciation, and ecological drift driving community and } \\
\text { metacommunity composition over time, based on an assumption of ecological equivalence. }\end{array}$ \\
\hline Neutral model & $\begin{array}{l}\text { Mechanistic model of individual life, death, reproduction, and dispersal events, under the } \\
\text { assumption of per capita fitness equivalence. These basic stochastic events collectively yield } \\
\text { neutral dynamics. }\end{array}$ \\
\hline Strict ecological equivalence & $\begin{array}{l}\text { All of the individuals have identical prospects of living, dying, reproducing, and dispersing, } \\
\text { which implies that they have equal fitnesses. They can display distinct ecological } \\
\text { properties, but equalizing mechanisms then yield fitness equality (Figure 2a). }\end{array}$ \\
\hline $\begin{array}{l}\text { Emerging ecological } \\
\text { equivalence }\end{array}$ & $\begin{array}{l}\text { All of the populations of coexisting taxa show positive growth rates from low density, } \\
\text { meaning that there is no better competitor. Strict equivalence implies emerging } \\
\text { equivalence, but not the reverse. Nonequal individual fitness can be counterbalanced by } \\
\text { stabilizing mechanisms yielding emerging equivalence (Figures } 2 \mathrm{~b} \text { and } 2 \mathrm{c} \text { ). }\end{array}$ \\
\hline
\end{tabular}

not (MacArthur and Levins 1967). The word "cause" here purports to a view of a "difference maker" (Lewis 1973; Woodward 2003; Hall 2004; Menzies 2004; Waters 2007), namely, a cause is a difference (e.g., changing the value of an input variable) that makes a difference (e.g., changing the value of an output variable). In the case of nichebased species coexistence, the difference in ecological characteristics between two species makes a difference in resulting species relative abundances. A species is expected to become more abundant than other species in a local community if it displays some attributes conferring better abilities for survival and reproduction in the local environment (Shipley et al. 2006). The niche theory then provides an explanation based on determinate causal processes at the individual level (e.g., competition, predation, or mutualism). In population genetics, to be selected likewise means to be there because of a difference in individual fitness due to some genotypic attributes (Sober 1993; Abrams 2007; Brandon and Ramsey 2007; Dietrich and Millstein 2008). Therefore, in niche theory, the difference-between species, alleles, or genotypes-makes a difference upon the identity of species, alleles, or genotypes present at equilibrium, so that it provides causal relations in the sense of difference-making.
Conversely, neutral dynamics are not causal in the same sense that niche-based processes are. Even though the relative species abundance distributions can be determined by a given set of neutral parameters, such as the fundamental biodiversity number of Hubbell (2001), the species composition is variable and changes from a replicate neutral community to another. If species A realizes the peak of the abundance distribution, by definition no biological property of $\mathrm{A}$ is a reason for its dominance in a neutral community; which means that, if we design a replicate community and consider the resulting biodiversity pattern under the assumption of ecological equivalence, we will get a similar relative abundance distribution without A necessarily being a dominant species. The explanatory target of the neutral theory concerns the relative species abundance and resulting diversity patterns at local and regional scales. It explains these patterns in the sense that it provides a way to generate them while minimizing the appeal to the influence of numerous biological properties.

As a consequence, neutral and niche theories cannot be considered two rival hypotheses that would stand on the same level because their explanatory targets are partially different. A major reason for the success of neutral theory in community ecology 
is that it predicts realistic patterns of biodiversity, expressed via distributions of species abundances that were debated in macroecology for a long time, such as the logseries distribution (Fisher et al. 1943; Preston 1948; Bell 2001). This quest for general laws has long been centered on species diversity patterns (essentially $\mathrm{SAD}$ ), which do not integrate the nature of species biological differences. A basic requisite of neutral theory is that these patterns should be independent of species properties. However, if a niche model predicts a species diversity pattern, it may also explain why some species are more represented than others based on some biological properties (Grime 1998; Shipley et al. 2006). Therefore, other patterns of diversity based on species properties should help detect the alternative effect of niche-based processes. Trait-based perspectives to community ecology (McGill et al. 2006b) and ecophylogenetics (Mouquet et al. 2012) have conveyed novel tests of nichebased processes by analyzing the diversity of species traits instead of relying solely on species taxonomy and abundances. Although the neutral theory does not say where a specific species stands in the abundance distribution, this question could be answered by niche theory, since niche properties then explain the success of a specific species in a specific environment (Chase 2005). We will keep this clarification in mind while turning to a second step for a specification of what the ecological equivalence assumption can integrate.

\section{The Origin(s) of Ecological EQUivalence}

The neutral theory explains and predicts biodiversity patterns at community and/or regional scales, based on the assumption of ecological equivalence, also termed neutrality assumption. Basic per capita ecological equivalence is defined in a very broad sense by Hubbell: "I use neutral to describe the assumption of per capita ecological equivalence of all individuals of all species in a trophically defined community. This is a very unrestrictive and permissive definition of neutrality because it does not preclude inter- esting biology from happening or complex ecological interactions from taking place among individuals" (Hubbell 2001:6). The fuzzy limits clearly reflect the fact that the assumption represents some effective ecological equivalence. In the following, we will address how ecological equivalence so broadly defined can be reached in different ways, thus entailing different conceptions of neutrality. Quite independently of the neutralist perspective, Chesson (2000) proposed a general conceptual framework of diversity maintenance in ecological communities, linking individual fitness and niche properties to community-level patterns of coexistence. Here we will use this framework to explore and discuss the meaning of ecological equivalence. It is based on the long-term per capita population growth rate of a putative invader $i$, denoted as $\bar{r}_{i}$, which initially occurs at low density in a local community. Invasibility here represents the ability of a species to increase from low abundance in the presence of the resident species of the community. If there is resource limitation in a system with a resident $s$ and an invader $i$, the per capita population growth rate of $i$ is $\bar{r}_{i}=b_{i}\left(\frac{\mu_{i}}{b_{i}}-\frac{\mu_{s}}{b_{s}}\right)$, where $\mu$ represents the mean per capita growth rates of species $i$ and $s$ in the absence of resource limitation, and $b$ is the rate at which these per capita growth rates decrease as resources decrease (Chesson and Huntly 1997; Chesson 2000). $k$. = $\mu . / b .(.=i$ or $s)$ is then a measure of the relative average fitness of species at population level, such that a species with the largest $k$ will be the winning competitor. This basic model cannot lead to stable coexistence, as $\bar{r}_{i}$ will be positive for one species only.

Conversely, in a context of niche partitioning between coexisting species, the per capita population growth rate of $i$ can be written as $\bar{r}_{i}=b_{i}\left(k_{i}-k_{s}\right)+b_{i}(1-\rho) k_{s}$, where $\rho$ is a parameter of niche overlap of the two species, that is, the proportion of resources they both use. With $\rho<1$ (niches do not completely overlap), the growth rates can be positive for both species $i$ and $s$, and competitive exclusion by a top competitor 
can be avoided. The model can be generalized to multispecies assemblage, such as

$$
\bar{r}_{i}=b_{i}\left(k_{i}-\bar{k}\right)+\frac{b_{i}(1-\rho) D}{n-1},
$$

where $n$ is the number of species, $\bar{k}$ is the average fitness of resident species, and $D$ is a positive constant. Coexistence will be possible for a set of species $i$ when their $\bar{r}_{i}$ values are positive, and long-term coexistence will occur if the $\bar{r}_{\text {, values are close to }}$ 0 . This is a form of ecological equivalence expressed at the level of population dynamics, which differs from Hubbell's (2001) primary assumption of fitness equivalence of individuals. This population-level equivalence can be achieved either by reducing the magnitude of per capita fitness differences between species in the left term of Equation (1) (equalization; Figure 2a) or if processes overcome these differences in the second stabilizing term of Equation (1) (stabilization; Figures 2b and 2c; Adler et al. 2007). We will consider this formalism to explore the limits of the basic fitness equivalence assumption in the neutral theory (extent of equalizing mechanisms), and the robustness of its predictions when fitness equivalence is violated (extent of stabilizing mechanisms).

\section{EQUALIZING MECHANISMS}

Ecological equivalence has been captured by the concept of fitness invariance in the framework of Hubbell: "By fitness invariance I mean that there are different trade-off combinations of life-history traits that confer equivalent per capita relative fitnesses on the species exhibiting them" (Hubbell 2001:322). Even when ecological variation exists among individuals and species, the assumption holds whenever the lifetime survival and fecundity probabilities are invariant in mathematical expectation among individuals. Averaging out these differences amounts to equalizing fitness. Therefore, equivalence can stem from aggregated intrinsically nonneutral differences between organisms (Hubbell 2006; Doncaster 2009). Under Hubbell's definition of fitness invari- ance, the right term of Equation (1) is equal to 0 (no stabilization), and the left term must be kept close to 0 to allow long-term species coexistence. The model then provides an instance of neutral dynamics. As Adler et al. noted, "[n] eutral models are simply the special case where species have equivalent fitness and there are no stabilizing, niche-based processes" (Adler et al. 2007: 96). The theory is thus robust to trait variation among species regarding some aspects of their biology, as far as they result in similar levels of fitness (Figure 2a).

\section{STABILIZING MECHANISMS}

Conversely, several studies showed that patterns of community diversity quite similar to those predicted by the neutral theory can be found even when fitness is uneven across species (Chave et al. 2002; Allouche and Kadmon 2009; Doncaster 2009). In these cases, the fitness difference in the left term of Equation (1) is no longer equal to 0 and the model does not conform to the equivalence assumption as defined by Hubbell (2001). However, the stabilizing term of Equation (1) can still be large enough to overcome the effect of fitness differences. Specifically, the stabilizing term can be increased by niche differences between species, which allows species coexistence in communities at equilibrium (MacArthur and Levins 1967). In particular, niche differences between species make competition stronger within species than between species, so that the growth rate $r_{i}$ can decrease when population density of species $i$ increases (negative density dependence). Negative density dependence can, therefore, prevent competitive exclusion by maintaining the coexisting species at low density (Levine and HilleRisLambers 2009). Another factor contributing to the stabilizing term is differences in colonization abilities (Mouquet and Loreau 2003; Figure 2c). In this case, a less competitive species can survive in a community because more competitive species display lower colonizing ability, so that they cannot establish everywhere and occupy all of the communities at the same time (Hubbell 1979; Tilman 

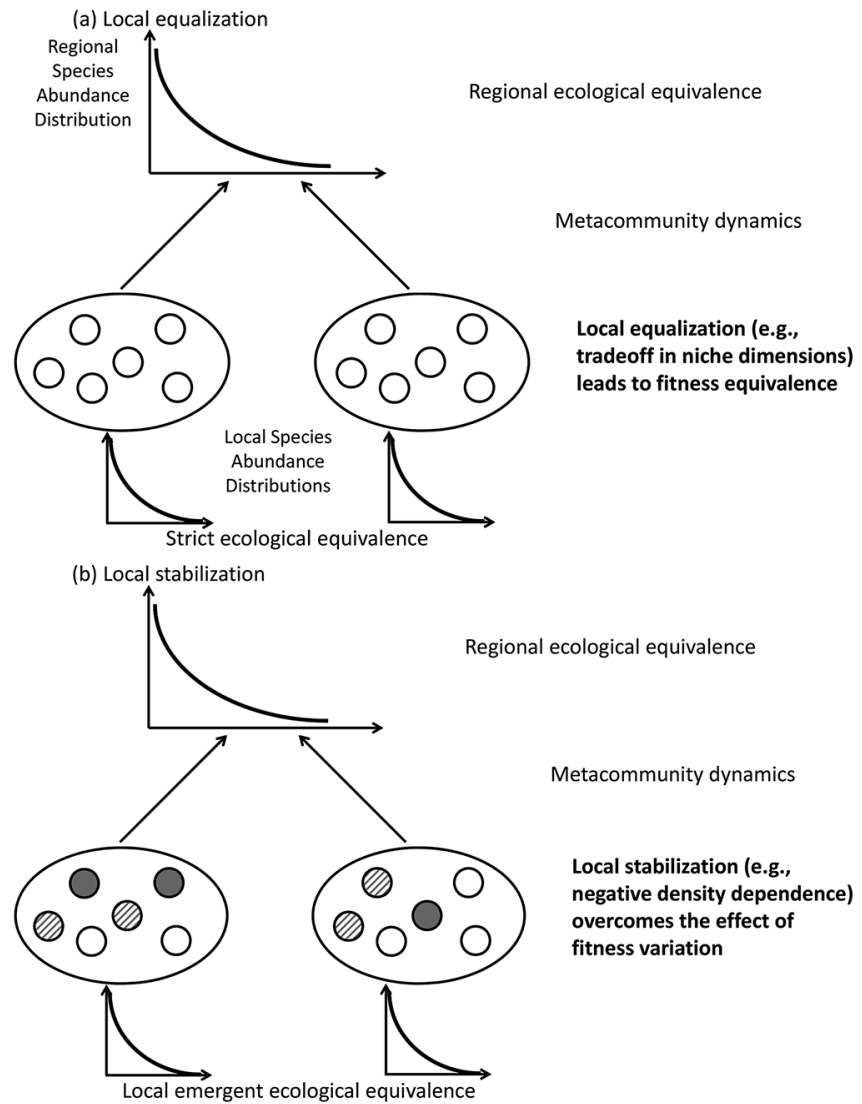

Metacommunity dynamics

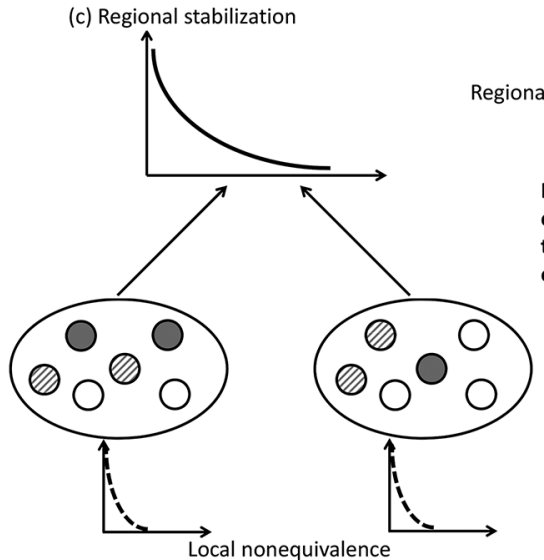

Local stabilization (e.g.,

negative density dependence)

overcomes the effect of

fitness variation

Regional stabilization (e.g.,

tradeoff) overcomes the effect

of fitness variation

Fitness differences influence

local community dynamics

Figure 2. Patterns and Spatial Scales of Ecological Equivalence

The ellipses represent local communities including a set of individuals (circles). The motif and the shading of the circles represent fitness variations between individuals. The three figures exemplify situations where ecological equivalence is expected at local and/or regional scales. The curves represent some putative species abundance distributions at local and regional scales, in which species abundances depend on their niche properties (dashed line) or not (solid line). The figures then basically stress when and how species abundances are not influenced by niche differences at local and regional scales. In the strong interpretation of neutral theory (a), effective fitness equivalence of individuals within communities leads to niche-independent dynamics. Variation in ecological characteristics among species is possible, insofar as they level out and yield 
1994). Coexistence of many species is thus possible within communities despite a competitive hierarchy (Mouquet and Loreau 2003). Here stabilization occurs in the overall set of communities connected by migration (the metacommunity), and can maintain species equivalence in the local community despite the asymmetry in species fitness.

\section{EMERGENCE OF ECOLOGICAL EQUIVALENCE}

Therefore, a per capita ecological equivalence can be reached via two ways in Chesson's framework. Doncaster (2009) introduced the concept of realized fitness to stress that "species must achieve ecological equivalence at their coexistence equilibrium, which is defined by equal realised fitness for all" (Doncaster 2009:2). Therefore, various mechanisms can make the global state of a community close to ecological equivalence through the stabilization and equalization terms of Equation (1). The role of equalization and stabilization is central to the distinction between the "weak" and "strong" interpretations of neutral theory. The strong interpretation is a mechanistic view of the neutral theory, where the fitness equivalence must actually be met at the individual level, with or without the influence of equalizing mechanisms. In this case, equivalence is explanatory of the processes that yield the biodiversity patterns. Conversely, "the weak version recognizes that the... [neutral theory] is capable of generating patterns that resemble those arising from survey data, without acknowledging that it correctly identifies the underlying mechanism responsible for generating these patterns" (Bell 2001:2418, emphasis added). Therefore, ecological equivalence entails neutral dynamics in the strong inter- pretation, while it is a consequence of neutral and possibly nonneutral dynamics in the weak interpretation of the theory. Here stabilization can counterbalance the influence of fitness differences and generate patterns of relative abundances close to that of neutral models (Chave et al. 2002; Allouche and Kadmon 2009; Doncaster 2009). In this case, the pattern is considered to be neutral in the weak interpretation, but the dynamics are nonneutral in the sense of the strong interpretation. It entails that a basic neutral model of speciation, migration, and drift does not necessarily represent actual dynamics in the weak interpretation.

A central role in the weak interpretation is granted to dispersal limitation, which can act as both an equalizing and a stabilizing factor (Holyoak and Loreau 2006:1373). It is equalizing because dispersal limitation limits the number of competitors coexisting at a given time and hence the extent of effective fitness differences (Hurtt and $\mathrm{Pa}$ cala 1995). It is stabilizing because locally dispersed propagules tend to generate spatial clustering of populations, which increases intraspecific competition compared to interspecific competition. In addition, dispersal couples local and regional species dynamics and requires moving from the individual-level conception of equivalence to a larger-scale emerging conception, as understood in the weak interpretation. Acknowledging the pervasive effect of dispersal limitation in ecological communities is, therefore, a key to understanding the relevance of ecological equivalence when explaining patterns of biodiversity from local to regional scale.

In this context we can specify the reason why the term process does not mean the same thing in neutral and niche-based models. On one hand, niche-based models address the

equivalent fitness. In a broader perspective of emerging ecological equivalence, as understood in the weak interpretation of neutral theory, there is no effect of biological differences on species relative abundances thanks to stabilizing mechanisms. In the case of local stabilizing mechanisms (b), fitness differences are counterbalanced by mechanisms such as negative frequency dependence within communities, which prevent the most competitive species to dominate. In the case of regional stabilizing mechanisms (c), fitness differences within local communities are counterbalanced by the dynamics between communities as, e.g., when there is a competition-colonization tradeoff. Stabilization allows local and/or regional ecological equivalence despite actual fitness differences between individuals (variation of motif and shading of individuals). 
determinate effect of elementary ecological processes based on specific, individual-level biological differences. These differences constitute the basic ecological nonequivalence. On the other hand, neutral theory represents stochastic biodiversity dynamics based on some species ecological equivalence, but it does not preclude a role of niche-based differences in yielding this ecological equivalence. Ecological equivalence can itself be the result of different processes-here, based on equalizing and stabilizing mechanisms. Therefore, when one actually wants to talk of "neutral processes," there is an equivocation: "the neutral processes" either mean a set of equalizing and stabilizing mechanisms yielding ecological equivalence, or the dynamics occurring under the condition of ecological equivalence. With respect to this equivocation, Doncaster wrote that "neutral patterns need not imply neutral processes" (Doncaster 2009:2).

Thus we have used the Chesson's framework to address the nature of ecological equivalence from the individual to population level. Because individual niche differences can play a role in stabilization, the way ecological equivalence is reached in this framework does not necessarily conform to the patterns predicted by neutral models considering strict equivalence at the individual level. But whatever the mathematical nature of the pattern is, any ecological equivalence emerging from equilization and stabilization prevents a determinate influence of niche differences on the species relative abundances. In this regard, it basically emphasizes the role of unspecified interactions and stochastic dynamics, which conforms to the nature of neutral theory (see the section, An Explanatory Theory of Unspecified Individual Interactions). The focus on emerging ecological equivalence thus allows extending the scope of original neutral models while retaining their epistemic specificity. Given that the processes considered at the source of ecological equivalence may not occur at the same scale, this leaves open the issue of the scale at which ecological equivalence can occur, a question that we now address.

\section{What IS The Scale of ECOlOGical EQUivalence?}

In order to clarify at which spatial and temporal scale ecological equivalence can occur, one needs to characterize the role of species ecological attributes for their persistence at nested spatial scales. A traditional top-down scheme of niche-based processes represents successive ecological filters determining how individuals from a regional source may establish and survive in a local community (Lortie et al. 2004; Cornwell and Ackerly 2009; Figure 3 left). However, such a top-down approach to niche-based processes is challenged by the idea of ecological equivalence, which can emerge from stabilizing and equalizing processes operating at a finer spatial scale (Figure 3 right). In addition, Chesson (2000) highlighted that the balance of equalizing and stabilizing mechanisms can change according to the spatial and temporal scale. Therefore, Holyoak and Loreau stressed that ecological equivalence "occurs because species that have spatial or temporal niche partitioning become equivalent in their competitive abilities at some spatial or temporal scales" (Holyoak and Loreau 2006:1370). Several hypotheses exist regarding the scales at which equalizing and stabilizing mechanisms can yield ecological equivalence:

Local-scale equalization (Figure 2a). Hubbell (2001) and Leibold and McPeek (2006) proposed that local guilds of functionally equivalent species are included in a matrix of heterogeneous habitats inhabited by other guilds (see also Leibold 2008). In this case, niche variation can exist between individuals of distinct communities, while the individuals within local communities are ecologically equivalent (Walker 2007). The ancient concept of ecological guild and the modern notion of a functional group represent such hierarchy of ecologically similar organisms embedded in dissimilar groups (Wilson 1999). A point of reference for this theory is the guilds of canopy tree species in wet evergreen tropical forests: many species, often more than 100 species in a 1 ha patch of forest, appear to be functionally equivalent 


\section{Community ecology upside-down}

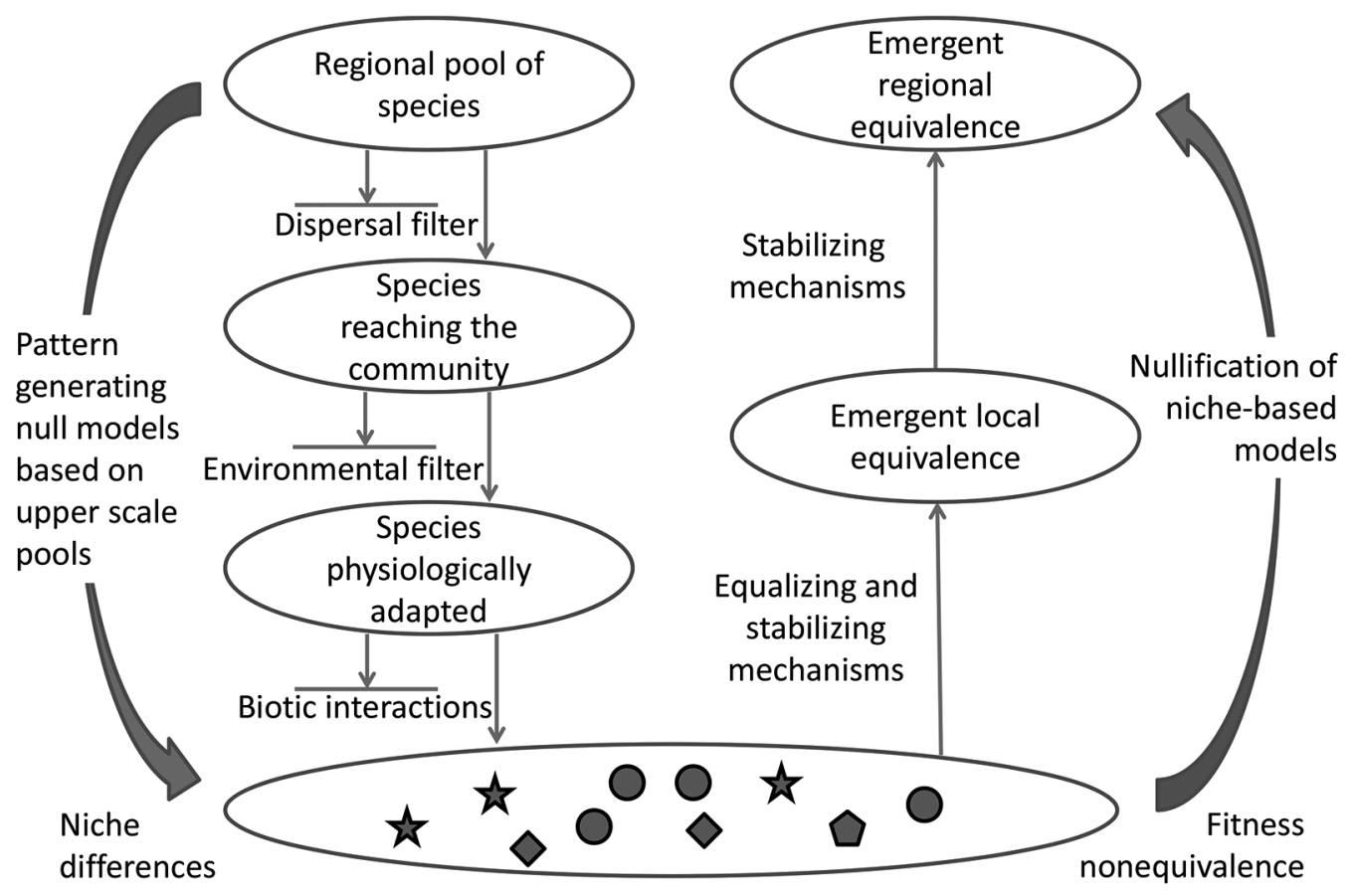

Local community including individuals with varying characteristics

Figure 3. Multiscale Biodiversity Dynamics and Hypothesis Testing

Contrasting representation and hypothesis testing of biodiversity dynamics in a top-down framework of niche-based ecological filters from the regional scale (left, adapted from Lortie et al. 2004), and in a bottom-up framework from individual dynamics to emerging patterns of ecological equivalence (right). In both cases, the varying ecological properties of local individuals (different symbols in the community ellipse) entail niche differences. On the left, the diversity of the properties observed in the community is the result of filtering processes depending on niche differences and selection of successful individuals from upper-level pools. On the right, tradeoff in niche dimensions can result in fitness equalization, or niche differences can overcome the effect of competition to yield emergent neutral patterns of community structure (local ecological equivalence). Larger scale mechanisms of stabilization (e.g., source-sink metacommunity dynamics) can further generate neutral patterns of biodiversity at the regional scale, even if niche-based processes predominate in local communities (see Figure 2). These contrasting frameworks involve different approaches for hypothesis testing. In the top-down framework, the uppermost-level pool is given and random communities are generated by assuming the absence of filters (pattern-generating null models). In the bottom-up framework, mechanistic models of niche-based and neutral dynamics are compared to their neutral counterparts without niche difference (nullification), and their predictions are tested at local and regional scales.

and display a neutral pattern of relative species abundances at very local scale. The spatial extent of habitats occupied by guilds varies according to the nature and function of the organisms (e.g., coral reef communities, mycorrhizal networks in soil). In this regard, ecological equivalence is restricted to a homogenous environmental context where coexisting species display similar prospects of living, reproducing, and dispersing, while environmental variation selects different compositions in separate communities. Dispersal limitation contributes to local equalization by limiting the probability that many 
competitors are found in the community at the same time (Hurtt and Pacala 1995). In an evolutionary perspective, the interplay of neutral and niche-based dynamics can yield the emergence of distinct guilds along environmental gradients (Scheffer and van Nes 2006; Vergnon et al. 2009).

Local-scale stabilization (Figure 2b). Local stabilizing mechanisms can prevent competitive dominance and maintain species-rich local communities. This is the core idea of the negative density dependence model, which predicts that the competitive advantage of a species decreases as its density increases because it is penalized by stronger intraspecific competition. The underlying mechanism may be related to limited resources (Levine and HilleRisLambers 2009), including limited pollinator availability (Chesson and Warner 1981; Gigord et al. 2001) or to species-specific parasites or predators (Louda et al. 1990; Hatcher et al. 2006; Johnson et al. 2012). As mentioned above, dispersal limitation also contributes to negative density dependence and local stabilization because clusters of individuals of the same species are formed close to the parents and increase intraspecific competition (Holyoak and Loreau 2006:1373).

Large-scale stabilization (Figure 2c). Much research in ecology still emphasizes that variation in local performance relates to niche differences between coexisting species (Grime 1998; Shipley et al. 2006). In situations where such nonneutral processes dominate locally, niche variation across species can still average out across communities dispatched in heterogeneous habitats, leading to a larger-scale pattern of ecological equivalence (Lavin et al. 2004; Pueyo 2006; Muneepeerakul et al. 2008). Dispersal limitation acts as a mechanism of large-scale stabilization by avoiding the predominance of better competitors or better colonizers across communities (Mouquet and Loreau 2003). This form of stabilization can create neutral patterns of biodiversity at the regional scale, but not locally.

Therefore, addressing niche-based and neutral dynamics is fundamentally contingent upon the spatial and temporal scales of interest (Chase 2014; Garzon-Lopez et al.
2014). A critical issue here is to characterize ecological objects at a level (e.g., by delineating guilds) and a scale (local or regional) that comply or not with an equivalence assumption. In addition, considering the way ecological equivalence and niche-based dynamics are intertwined across scales uncovers a tension between topdown (from regional to local) and bottomup (from local to regional) approaches to biodiversity dynamics (Figure 3). To this extent, the question of how to handle ecological equivalence connects to Ricklefs' (2008) challenge about community ecology. He argued that working at the community level is not appropriate because it does not take into account the influence of regional dynamics. The way niche-based and neutral dynamics drive regional biodiversity in turn influence the dynamics of local communities receiving immigrants (Mouquet and Loreau 2003). In our case, we highlight the potential for confusion in any discussion of the status of the neutral theory that implicitly focuses on the local scale.

Furthermore, the question of scale concerns not only community assembly in geographic space, but also evolution in multidimensional niche space. Scheffer and van Nes (2006) predicted that guilds of equivalent species can evolutionarily emerge in distinct regions of niche space, as a result of both niche-based and neutral processes. As a consequence, " $[\mathrm{w}]$ hen considering pairs of competitors, or species-poor assemblages, competitive divergence is expected, but when considering entire, species-rich communities, convergence among subsets of the community can generate sets of nearly competitively equivalent species" (Holt 2006:532).

Up to this point we have analyzed differences proper to the explanatory structure of neutral theory in ecology: first, the explanatory nature and the predictions of neutral models versus niche models and, second, the processes yielding ecological equivalence either locally or regionally (Figures 2 and 3). The role of equalizing and stabilizing mechanisms is central here, and models of ecological equivalence can extend beyond neutral models based on individual fitness equivalence (see the section, 
Emergence of Ecological Equivalence). In what follows, we discuss the status of neutral theory regarding its role as a possible null hypothesis or a parsimonious baseline for community and metacommunity ecology in such a broad perspective of ecological equivalence.

\section{Is the NeUtral TheOry a Null or a Parsimonious Hypothesis?}

The success of the neutral theory since Hubbell (2001) has primarily relied on its heuristic ability to account for some patterns of diversity, even though these patterns had been interpreted as niche-driven for decades. Based on the fit of species abundance distributions, some authors compared the predictive ability of neutral versus niche-based models, yielding conflicting results and much debate (Chave et al. 2002; McGill 2003). A critical point is whether such comparison allows concluding in favor of one or the other theory. Therefore, much subsequent research turned to define neutral models as a basis for hypothesis testing (Bell 2001; Gotelli and McGill 2006). Now that we have circumscribed the explanatory specificity of neutral theory and of the central equivalence assumption in a scaledependent framework of equalizing and stabilizing mechanisms, we aim to clarify the status of the neutral theory for hypothesis testing after one decade of conflicting views. In this regard, we will investigate the meaning of the neutralist claim that neutral models explain biodiversity patterns (Bell 2001; Hubbell 2001), against the idea that neutral models should rather be used as null hypotheses for understanding these patterns (Holyoak et al. 2006; McGill et al. 2006a).

\section{CONFLICTING CONCEPTIONS OF THE NULL HYPOTHESIS}

Beyond the context of neutral theory, two diverging conceptions exist in ecology on what a null hypothesis is:

Generation of null patterns. Gotelli and Graves (1996) promoted an instrumentalist treatment of the null hypothesis based on pattern-generating null models. These models perform "randomization of ecological data or random sampling from a known or imagined distribution. The null model is designed with respect to some ecological or evolutionary process of interest. Certain elements of the data are held constant, and others are allowed varying stochastically to create new assemblage patterns. The randomization is designed to produce a pattern that would be expected in the absence of a particular ecological mechanism" (Gotelli and Graves 1996:3-4). Emphasis is put here on designing a randomization procedure that generates a variety of virtual communities complying with a null hypothesis, under which some processes are not involved. The alternative hypothesis hence states that these neglected processes are actually involved, and the null hypothesis is rejected when the observed patterns significantly deviate from the randomized communities, provided that the empirical data are relevant enough to allow discernment. This approach is closely related to the top-down conception of ecological filters (Figure 3 left), where the constraints represent a regional background context from which we assemble null communities by using a randomization scheme. There has been much debate on how to design appropriate null models (Gotelli and Entsminger 2001, 2003; Gotelli and Ulrich 2012; Ulrich and Gotelli 2013). In order to detect the effect of nichebased processes in the alternative hypothesis, classical randomization schemes shuffle species biological attributes or community members under the null hypothesis that the distribution and abundances of species is independent of local environmental conditions and species attributes. A major issue is the nature of the processes represented by such a randomization scheme: Do randomization procedures comply with a specific model of community dynamics? And, if not, should community dynamics be simulated directly?

Nullification of model parameters. In the second sense, a "null hypothesis" represents the influence of a set of processes but nullifies their parameters. To understand this, think of a process governed by several parameters, such that the null instance of 
the process is obtained when all the parameters are set to 0 . Whether or not setting parameters to 0 in the model influences the ability to predict patterns similar to the observed one allows falsifying the null hypothesis, based on a statistical criterion of model comparison (BurnhamandAnderson 2002). Contrarily to the pattern-generation conception, the nullification conception of null hypothesis represents explicit community dynamics.

To what extent can the neutral theory be employed as a null hypothesis (Holyoak et al. 2006; McGill et al. 2006a)? On one hand, the equivalence assumption can be considered the nullification kind, such as the relative fitness parameters $w_{i}$ of all species are set to 0 . If you consider Equation (1), the null hypothesis would be then given by nullification of fitness differences only (equalization), which means that the terms from the stabilizing function are 0 : it is the interpretation given by Adler et al. when they see neutrality as a "special case where ... there are no stabilizing, nichebased processes" (Adler et al. 2007:96). Thus, the nullification approach resorts to the strong interpretation of the neutral theory. Under this interpretation, we see how the neutral model can be a null hypothesis: instantiating the random processes that take place when parameters ruling the influence of any biological difference are set to 0 . In this regard, the main motivation of "nearly neutral models" (Ohta 1992; Zhou and Zhang 2008; Noble et al. 2011) is to provide an alternative model of fitness differences such that individual equivalence is obtained by simply constraining the parameters. In addition, because the null hypothesis explicitly considers no influence of biological differences, any ecological equivalence emerging due to stabilizing mechanisms in fact pertains to the alternative hypothesis.

On the other hand, we have underlined that the weak interpretation of neutrality is based on emerging neutral patterns, whatever the fine-scale dynamics are. We have integrated in this perspective patterns of ecological equivalence emerging at the population level in Chesson's framework (see the section, Emergence of Ecological Equivalence). In any case, emerging ecological equivalence constitutes a null hypothesis of the pattern-generation kind, resulting from both neutral and nonneutral dynamics through equalization and stabilization. In this conception, the role of the neutral theory "is then restricted to providing the $a p$ propriate null hypothesis when evaluating patterns of abundance and diversity. Even this relatively modest role, however, involves revising the comparative approach to ecology" (Bell 2001:2418, emphasis added). Holt concurred: "In its 'weak' form, neutral theory at the very least provides the appropriate null model for evaluating patterns in comparative data sets" (Holt 2006:531, emphasis added). Since the weak interpretation resorts to neutral pattern generation, it implies that the null hypothesis here is not defined by parameter nullification. Even so, the weak interpretation still does not comply with a randomization way of generating null patterns. Patterns resulting from dispersal and local birth-death dynamics are indeed sensibly different from that of shuffled compositions, as Bell underlined: "[s] tatistical null hypotheses based on randomization are not appropriate for evaluating ecological patterns that stem from species distributions, because local dispersal readily gives rise to spatial patterns" (Bell 2001:2418). Therefore, simulation of emerging ecological equivalence should be preferred to randomization of community composition. A difficulty of such a pattern-generating model is that it needs to estimate the parameters of the dynamics from the data (Gotelli and McGill 2006).

In the perspective of using neutral theory as a null hypothesis, we have related emerging ecological equivalence, and thus the weak interpretation of the theory, to the first kind of null hypothesis, i.e., pattern generating, and the strong interpretation of neutral theory, which assumes strict equivalence at individual level to the second kind of null hypothesis, i.e., parameter nullification. In both cases, a critical issue is whether a model of neutral dynamics provides a well-defined null hypothesis. Specifically, we need to clarify what role we 
would expect for niche differences in null and alternative hypotheses. Several authors have claimed that the hypotheticodeductive approach should begin with the examination of some maximally parsimonious model of community dynamics by eliminating nonexplanatory components related to an additional influence of niche differences. Holyoak and Loreau emphasized that we have the choice "of whether to appeal to parsimony and select a [neutral community model] ... or to accept a more complex niche model" (Holyoak and Loreau 2006:1372). But, as noted above, ecological equivalence can emerge even when niche-based processes influence individual dynamics because of equalizing and stabilizing mechanisms (Figure 2). In this regard, emerging ecological equivalence is not a well-specified null hypothesis when the alternative hypothesis should identify a role for specified niche dimensions.

In addition, we have underlined that the predominance of neutral and niche-based processes is basically scale-dependent (see the section, What is the Scale of Ecological Equivalence?). It is then crucial to keep in mind that null hypotheses must be defined in a certain context and at a certain level. Hence, added to the alternatives of pattern generation and nullification in hypothesis testing, we have to consider the level and scale at which neutrality is envisaged. Conflicting takes on the neutral theory then reflect conflicting views on whether a "strong" neutral model would be an explanation for patterns extended from individual-level ecological equivalence or a "weak" model would provide an emergent null reference against which to detect a large-scale imprint of niche-based processes (Araújo and Rozenfeld 2014). In this regard, aknowledging emerging ecological equivalence is required to address the scaling of niche-based processes from the scale of individuals bearing distinct biological attributes to larger-scale patterns of biodiversity.

The focus thus shifts from testing the neutral theory against a single niche-based alternative to testing ecological equivalence across scales. In the strong interpretation, a hierarchy of alternative hypotheses is needed to address the extent of the influence of stabilization and niche differences at multiple scales in space and time. The role of stabilizing processes must then be identified in the alternative hypotheses as, for instance, we expect that density-dependent effects would generate nonrandom spatial distributions of conspecific and heterospecific individuals (Comita et al. 2010). In the context of the weak interpretation, complying with a pattern of neutral dynamics at a given scale entails that niche differences do not contribute to explain it: it is a contraposition of the hypothesis that niche differences should influence biodiversity patterns, but not a validation of the hypothesis that everything is neutral. Therefore, the two approaches are not incompatible, but involve a different specification of the null hypothesis. As we will see below, the way the hypotheses are specified determines the conclusion to reach from their acceptation or rejection.

\section{FALSIFICATION VERSUS PARSIMONY}

We have seen above that neutral theory can be used as a null reference, but also that the nature of the null and alternative hypotheses is scale-dependent and differs between the weak and stong interpretations. Yet, apart from these distinctions, a more general methodological issue raised by neutral models in ecology is whether we should concentrate on rejecting a null neutral hypothesis (falsification), or we could accept it if the predicted patterns are consistent with observed ones (parsimony):

Falsification. In the hypothetico-deductive approach, the fact that the null hypothesis is not falsified does not mean that it is true. As Rosindell et al. stated, "[i]t does not follow that species-specific qualities are absent in the real world; instead, it suggests that their effects do not penetrate the foggy lens of the summary statistics being studied. This might explain how a neutral model can effectively fit data from a non-neutral world" (Rosindell et al. 2011:342). Biodiversity patterns may not be informative enough to allow discriminating the contributions of neutral and nonneutral dynamics, as they could in- 
differently be generated by a niche-based or by a neutral model (Chave et al. 2002; Purves and Pacala 2005; Chisholm and Pacala 2010). Then using a neutral model as a null hypothesis implies that only rejection of the null hypothesis makes sense, that is, we know in this case for sure that something else than this neutral model should be proposed for explaining the pattern of interest (McGill et al. 2006a).

Parsimony. On the other hand, a central concern in biology is to favor parsimonious explanations (Occam's razor; Sober 1981). In this regard, if two models can explain a given pattern, one should keep the most parsimonious one. A great deal of research in ecology is devoted to investigating alternative explanatory models according to a balance between their goodness of fit and their complexity (Burnham and Anderson 2002), given that too complex models may pick out too much noise in the data. Then, if both a niche-based and a neutral model can explain an observed pattern equally well, the neutral model would be the best choice as it provides a more parsimonious explanation (Holyoak and Loreau 2006). In the tradition of macroecology and biogeography, the neutral theory thus provides the most simple explanation of large-scale diversity patterns (Bell 2001). This parsimony criterion has led some researchers to conclude that neutral theory predicts robust emerging patterns and is a relevant basis to assess the pervasive influence of dispersal limitation at large spatial scales (Leigh 2007). If the neutral theory is a parsimonious hypothesis of biodiversity dynamics, it is not to be falsified, on the contrary, it is a baseline from which to start.

\section{FROM PARSIMONY TO INTEGRATION:} BEYOND THE NICHE-NEUTRAL DIVIDE

A key argument for using neutral theory as a parsimonious baseline is the generality of the basic mechanisms. We have mentioned that dispersal limitation is both a key equalizing factor determining the equilibrium composition of neutral communities in the strong sense, and a stabilizing factor allowing biodiversity to stay close to neutral- ity locally and regionally despite differences in competitive and dispersal between species. Indeed, no organism can disperse instantaneously and homogeneously across a whole region. Holt (2006:531) thus underlined that dispersal limitation is pervasive in communities and may account for most neutral dynamics and patterns found in species-rich ecosystems. Stochastic fluctuations of birth and death rates are also ubiquitous and influence species population dynamics because of finite-size effects (Lande et al. 2003). Therefore, a reasonable standpoint is that dispersal and population sizes of any species are axiomatically limited, so that neutral dynamics should be acknowledged everywhere, but still with a varying relative importance compared to niche-based processes (Gravel et al. 2006; Leibold and McPeek 2006; Munoz et al. 2014).

Acknowledging the pervasiveness of neutral dynamics ultimately leads to an integrative perspective, which forces one to overcome dualities in the interpretation of the neutral model as a null hypothesis, and in the acceptation or refutation of the theory. The recognition that stochastic processes of birth, death, and immigration are ubiquitous and can predominate in some contexts has led some authors to plead for a more comprehensive approach, merging into one general model the effects of both niche-based and neutral processes (Gravel et al. 2006; Holt 2006; Holyoak and Loreau 2006; Adler et al. 2007; Vellend 2010). The neutral theory then becomes a component of a more general theory. In the logic of model selection mentioned above, one can then conclude that niche-based processes contribute to some observed pattern if they improve the goodness-of-fit with a limited increase in complexity (Burnham and Anderson 2002). Such an approach aims at disentangling the signatures of neutral and niche-based processes from their combined effect (e.g., Adler et al. 2007; Doncaster 2009).

In this perspective, the neutral theory is no more a null hypothesis but a proper parsimonious hypothesis for explaining biodiversity. It holds that the mechanisms responsible for neutral biodiversity patterns are indeed 
of the sort Hubbell described (i.e., ecological drift, etc.), and a neutral model is accordingly a reference parsimonious model among a set of more complex models further incorporating the effect of niche differences. Along this line, neutral ecology according to Hubbell (2005) did what neutral molecular evolution did regarding evolutionary theory (Veuille 2000): it shifted the burden of proof to the extent that the patterns against which one will check whether selection is acting (e.g., when doing a McDonald-Kreitmann test on genomic sequences) are no longer the expected patterns under selection, but the patterns expected under the hypothesis of neutral dynamics alone. Therefore, the roles of neutral and of niche-based theories are actually not symmetrical, as neutral theory provides a baseline, parsimonious model against which to test more complex theories. Differences from the neutral predictions are then not a cause for rejecting a null hypothesis, but rather a call for complexifying the model by adding some parameters initially taken as null.

\section{Discussion}

We have identified several fundamental epistemic divides that explain conflicting takes on the role and use of neutral theory in ecology. The strong and weak interpretations of ecological equivalence differ in the way basic mechanisms or emergent patterns are considered, respectively. These interpretations relate to distinct conceptions of ecological equivalence as defining a null hypothesis, either as a nullification or a pattern-generating hypothesis, respectively. Furthermore, apart from the weakstrong distinction, considering the neutral theory as a null reference appeals to the question of whether the aim is to falsify a neutral model or rather to accept it as a parsimonious explanation when data match the predictions. Beyond such a dichotomy, we have stressed that the neutral theory is, by essence, a theory of pervasive and ubiquitous stochastic dynamics related to limited dispersal and population size. From this analysis of the neutral theory, we consider now what its place in ecological re- search is and what are the perspectives opened by this recent paradigm.

\section{SITUATION OF THE NEUTRAL \\ THEORY IN LEVINS' TRIANGLE}

Wennekes et al. (2012) claimed that the perspectives appropriate to niche and neutral theories are different, in the sense Levins (1966) famously distinguished between possible model-building strategies based on the fact that a strategy cannot simultaneously fulfill generality, precision, and realism as distinct epistemic values. Neutral theory would aim for generality and niche theory for realism, which means that they provide complementary rather than conflicting perspectives. In this viewpoint it is difficult to make sense of the claim that neutrality could be a null hypothesis-something Wennekes et al. (2012) acknowledged, calling it a "baseline" model rather than a null model. Hence they see the neutral theory as an explanation that philosophically can be seen in an instrumentalist perspective.

In the view defended here, it is the very interpretation of ecological equivalence, as well as the perspective that the research strategy adopts on it, which determines the status of neutral theory. Whereas Wennekes et al. (2012) focused on a global opposition between realism and generality, we have insisted on the level- and scale-dependence of neutral and niche-based explanations. Understanding the stabilizing mechanisms that yield ecological equivalence in a community pertains to realism, whereas taking ecological equivalence as a macroscopic emergent property may illuminate the reasons for very general patterns in nature. These are two possible positions in Levins' scheme, but according to the fine-grained distinctions we have drawn between the strong and weak interpretations, the variety of epistemic distinctions between neutral and niche theories may increase. Finally, we have highlighted that neutrality can either emerge regionally from stabilizing mechanisms in the metacommunity, or locally in the community from equalizing and stabilizing mechanisms (Figure 2): hence in order to compare niche and neutral theories, the degree of generality 
of models seems to be less epistemically relevant than the status of scales.

\section{ECOLOGICAL EQUIVALENCE ACROSS SCALES}

To wrap up the view of the epistemological status of neutral theory and ecological equivalence proposed throughout the paper, let us recall our previous claims. First, there is a distinction between ecological equivalence as a cause in the strong interpretation of neutral theory, and as an emerging pattern in the weak interpretation (see the section, The Origin(s) of Ecological Equivalence). Second, neutral and niche-based processes are entangled over a hierarchy of spatial and temporal scales, and yield emerging equivalence at multiple scales (see the section, What is the Scale of Ecological Equivalence?). Third, neutral theory is a theory of ubiquitous stochastic dynamics of birth, death, dispersal limitation, and regional speciation. This implies that neutral theory considers basic components of actual biodiversity dynamics and, as such, it is a component of a more integrative theory (see the section, Is the Neutral Theory a Null or Parsimonious Hypothesis?). From these claims, a major challenge for modern ecology is to go beyond the niche and neutral divide, and to consider the nature and consequences of ecological equivalence. We should also go beyond the divide of the weak and strong interpretations, which should be bypassed by a focus upon integrating ecological equivalence in parsimonious models.

The significance of stabilizing mechanisms leading to emerging ecological equivalence depends on the way the influence of individual biological attributes on one hand, and of stochastic dynamics on the other hand, propagates across scales to shape macroscopic patterns. As both an equalizing and stabilizing mechanism, dispersal limitation is a central aspect of this multiscale perspective on ecological equivalence. Because most organisms are dispersal-limited, emerging equivalence is expected to be pervasive in ecological systems, not only when fitness equivalence occurs in local communities, but also via a combination of competition and dispersion leading to larger-scale stabilization, as in the model by Mouquet and Loreau (2003). In this regard, depending on the role of the stabilizing mechanisms, a neutral model based on strict individual ecological equivalence may not provide appropriate expectations of the species relative abundances. In contrast, the concept of emerging ecological equivalence goes beyond a particular neutral model, and concerns the fact that biological attributes do not make a difference in the relative abundances at local and/or regional scales. When authors say that there might be several ways toward neutral patterns, that in other words one can have neutral patterns with nonneutral processes (e.g., Doncaster 2009:3), they talk about this second version of a neutral model, i.e., more generally, they address the status of neutrality as an attractor for many processes taking place at several scales in space and time. We must move then from a neutral theory of stochastic individual dynamics to a broader theory of ecological equivalence across scales.

As such, a core aspect of a theory of ecological equivalence is that biological differences between species are not explanatory at all: hence we do not have here a causal explanation in the sense of difference making, as emphasized previously (in the section, An Explanatory Theory of Unspecified Individual Interactions), and no genuine causal process. Then in a perspective of emerging ecological equivalence, differences between species do not make a difference, but for a reason other than strict fitness equivalence: here, the differences play a role in stabilizing mechanisms so that, at an emergent level, they no longer make a difference. This aspect may be frustrating for ecologists who long aimed at finding causal pathways of niche-based dynamics propagating over scales. However, addressing ecological equivalence is crucial to understand whether and how biological differences matter to explain emergent patterns, because these emergent patterns in turn influence the availability of immigrants in regional pools of species (Mouquet and Loreau 2003; Lessard et al. 2012). 
In such an integrative perspective, neutral theory does not need to be proved or disproved. It is a parsimonious baseline model from which to build any refined model of biological interactions in order to understand the nature and extent of equivalence and nonequivalence in ecological systems.

\section{Conclusions}

First, characterizing the neutral theory as a null hypothesis (or not) seems too broad a characterization to capture what is epistemologically at stake when using neutral models. Mechanisms of species coexistence are multiple and nested in spatial scales and in niche dimensions, each of them defined by some salient processes, and these scales are at the same time decoupled and interacting (Figures 2 and 3). This was captured through the idea that the contribution of stabilizing and equalizing mechanisms to neutral patterns of biodiversity depends on spatial scale (see the section, What is the Scale of Ecological Equivalence?). Here "neutrality" includes many more models than the neutral theory sensu Hubbell (2001). In this sense, ecological equivalence is not a null hypothesis on the absence of niche-based processes, since it may explicitly refer to an entangled set of generating processes, including niche-based ones.

Second, therefore, the neutral theory encompasses, as explanatory, the processes that are involved in establishing a resulting or emerging ecological equivalence (see the section, An Explanatory Theory of Unspecified Individual Interactions). To this extent it is no longer the case that biological differences (between species) make no difference, amounting to a noncausal explanation-but these differences result in a pattern of no difference that can itself be explanatory of biodiversity. Therefore, we have to acknowledge that neutrality is not so neutral since it encompasses more than individual ecological equivalence. This extension and, at the same time, the weakening of the original neutral theory parallels the fate of the neutral theory in molecular evolution, which gave rise to a more explanatory, powerful, and encompassing "nearly neutral" theory with relaxed assumptions on fitness equality (Ohta 1992). The controversies and developments that we have reviewed in this paper seem to attest that such a move has also occurred in community ecology.

Third, the necessity of an integrative and multiscale framework of ecological equivalence exposes the fact that niche-based processes cannot provide an exclusive causal explanation of biodiversity dynamics in space and time. Community ecology has undergone a profound paradigm shift with neutral theory, not only by providing a robust theory based on ecological equivalence, but also by connecting local ecological dynamics to regional biogeographical and evolutionary dynamics. Beyond the neutral-niche divide, a crucial role of a theory of ecological equivalence will be to solve the tension between the bottom-up and top-down perspectives on this relationship-as they were described in the section, What is the Scale of Ecological Equivalence? and illustrated in Figure 3-and to show how emergent patterns of biodiversity can in turn influence large-scale biogeographical and evolutionary dynamics. This would make ecological equivalence the cornerstone of a comprehensive theory of the emergence and regulation of biodiversity dynamics at multiple scales. In this regard, it will no longer be a phenomenological theory of emergent patterns, but a theory of the explanatory significance of ecological equivalence.

Finally, future research should help understand and predict the robustness and resilience of biodiversity dynamics in the face of ongoing environmental changes. In this regard, the urgent need for predictive ecology requires taking into account the mechanisms coupling local and largerscale biodiversity dynamics (Mouquet et al. 2015). By considering the influence of equalizing and stabilizing mechanisms in space and time, multiscale modeling of neutral and niche-based dynamics should help forecast the rate and extent of biodiversity changes and the possible cascades of environmental alterations. 
ACKNOWLEDGMENTS

We warmly thank Pierre Couteron, Michael Dietrich, Sébastien Dutreuil, John Huss, Michael Strevens,
Cyrille Violle, the editors, and the two reviewers for their insightful comments and suggestions. This work is part of the project Explabio ANR-13-BSH3-0007.

\section{REFERENCES}

Abrams M. 2007. How do natural selection and random drift interact? Philosophy of Science 74:666-679.

Adler P. B., HilleRisLambers J., Levine J. M. 2007. A niche for neutrality. Ecology Letters 10:95-104.

Allouche O., Kadmon R. 2009. A general framework for neutral models of community dynamics. Ecology Letters 12:1287-1297.

Araújo M. B., Rozenfeld A. 2014. The geographic scaling of biotic interactions. Ecography 37:406-415.

Bell G. 2001. Neutral macroecology. Science 293:24132418.

Brandon R. N., Ramsey G. 2007. What's wrong with the emergentist statistical interpretation of natural selection and random drift? Pages 66-84 in The Cambridge Companion to the Philosophy of Biology, edited by D. L. Hull and M. Ruse. Cambridge (United Kingdom): Cambridge University Press.

Burnham K. P., Anderson D. R. 2002. Model Selection and Multimodel Inference: A Practical Information-Theoretic Approach. Second Edition. New York: Springer.

Chase J. M. 2005. Towards a really unified theory for metacommunities. Functional Ecology 19:182-186.

Chase J. M. 2014. Spatial scale resolves the niche versus neutral theory debate. Journal of Vegetation Science 25:319-322.

Chave J. 2004. Neutral theory and community ecology. Ecology Letters 7:241-253.

Chave J., Leigh E. G. Jr. 2002. A spatially explicit neutral model of $\beta$-diversity in tropical forests. Theoretical Population Biology 62:153-168.

Chave J., Muller-Landau H. C., Levin S. A. 2002. Comparing classical community models: theoretical consequences for patterns of diversity. American Naturalist 159:1-23.

Chave J., Alonso D., Etienne R. S. 2006. Comparing models of species abundance. Nature 441:E1.

Chesson P. 2000. Mechanisms of maintenance of species diversity. Annual Review of Ecology and Systematics 31:343-366.

Chesson P., Huntly N. 1997. The roles of harsh and fluctuating conditions in the dynamics of ecological communities. American Naturalist 150:519-553.

Chesson P. L., Warner R. R. 1981. Environmental variability promotes coexistence in lottery competitive systems. American Naturalist 117:923-943.

Chisholm R. A., Pacala S. W. 2010. Niche and neutral models predict asymptotically equivalent species abundance distributions in high-diversity ecological communities. Proceedings of the National
Academy of Sciences of the United States of America 107:15821-15825.

Comita L. S., Muller-Landau H. C., Aguilar S., Hubbell S. P. 2010. Asymmetric density dependence shapes species abundances in a tropical tree community. Science 329:330-332.

Cornwell W. K., Ackerly D. D. 2009. Community assembly and shifts in plant trait distributions across an environmental gradient in coastal California. Ecological Monographs 79:109-126.

Dietrich M. R., Millstein R. L. 2008. The role of causal processes in the neutral and nearly neutral theories. Philosophy of Science 75:548-559.

Doncaster C. P. 2009. Ecological equivalence: a realistic assumption for niche theory as a testable alternative to neutral theory. PLOS ONE 4:e7460.

Dowe P. 2009. Causal process theories. Pages 213-233 in The Oxford Handbook of Causation, edited by $\mathrm{H}$. Beebee, C. Hitchcock, and P. Menzies. Oxford (United Kingdom): Oxford University Press.

Ellis B. 1999. Causal powers and laws of nature. Pages 19-34 in Causation and Laws of Nature, edited by H. Sankey. Dordrecht (The Netherlands): Kluwer Academic Publishers.

Ewens W. J. 1972. The sampling theory of selectively neutral alleles. Theoretical Population Biology 3:87-112.

Fisher R. A., Corbet A. S., Williams C. B. 1943. The relation between the number of species and the number of individuals in a random sample from an animal population. Journal of Animal Ecology 12:42-58.

Friedman M. 1974. Explanation and scientific understanding. Journal of Philosophy 71:5-19.

Garzon-Lopez C. X., Jansen P. A., Bohlman S. A., Ordonez A., Olff H. 2014. Effects of sampling scale on patterns of habitat association in tropical trees. Journal of Vegetation Science 25:349-362.

Gewin V. 2006. Beyond neutrality-ecology finds its niche. PLOS Biology 4:e278.

Gigord L. D. B., Macnair M. R., Smithson A. 2001. Negative frequency-dependent selection maintains a dramatic flower color polymorphism in the rewardless orchid Dactylorhiza sambucina (L.) Soò. Proceedings of the National Academy of Sciences of the United States of America 98:6253-6255.

Gotelli N. J., Entsminger G. L. 2001. Swap and fill algorithms in null model analysis: rethinking the knight's tour. Oecologia 129:281-291. 
Gotelli N. J., Entsminger G. L. 2003. Swap algorithms in null model analysis. Ecology 84:532-535.

Gotelli N. J., Graves G. R. 1996. Null Models in Ecology. Washington (DC): Smithsonian Institution Press.

Gotelli N. J., McGill B. J. 2006. Null versus neutral models: what's the difference? Ecography 29:793-800.

Gotelli N. J., Ulrich W. 2012. Statistical challenges in null model analysis. Oikos 121:171-180.

Gravel D., Canham C. D., Beaudet M., Messier C. 2006. Reconciling niche and neutrality: the continuum hypothesis. Ecology Letters 9:399-409.

Grime J. P. 1998. Benefits of plant diversity to ecosystems: immediate, filter and founder effects. Journal of Ecology 86:902-910.

Hall N. 2004. Two concepts of causation. Pages 225276 in Causation and Counterfactuals, edited by J. Collins, N. Hall, and L. A. Paul. Cambridge (Massachusetts): MIT Press.

Hatcher M. J., Dick J. T. A., Dunn A. M. 2006. How parasites affect interactions between competitors and predators. Ecology Letters 9:1253-1271.

Holt R. D. 2006. Emergent neutrality. Trends in Ecology and Evolution 21:531-533.

Holyoak M., Loreau M. 2006. Reconciling empirical ecology with neutral community models. Ecology 87:1370-1377.

Holyoak M., Loreau M., Strong D. 2006. Neutral community ecology. Ecology 87:1368-1369.

Hubbell S. P. 1979. Tree dispersion, abundance, and diversity in a tropical dry forest. Science 203:12991309.

Hubbell S. P. 1997. A unified theory of biogeography and relative species abundance and its application to tropical rain forests and coral reefs. Coral Reefs 16:S9-S21.

Hubbell S. P. 2001. The Unified Neutral Theory of Biodiversity and Biogeography. Princeton (New Jersey): Princeton University Press.

Hubbell S. P. 2005. Neutral theory in community ecology and the hypothesis of functional equivalence. Functional Ecology 19:166-172.

Hubbell S. P. 2006. Neutral theory and the evolution of ecological equivalence. Ecology 87:1387-1398.

Hurtt G. C., Pacala S. W. 1995. The consequences of recruitment limitation: reconciling chance, history and competitive differences between plants. Journal of Theoretical Biology 176:1-12.

Johnson D. J., Beaulieu W. T., Bever J. D., Clay K. 2012. Conspecific negative density dependence and forest diversity. Science 336:904-907.

Kimura M. 1983. The Neutral Theory of Molecular Evolution. Cambridge (United Kingdom): Cambridge University Press.

Kitcher P. 1976. Explanation, conjunction, and unification. Journal of Philosophy 73:207-212.
Lande R., Engen S., Sæther B.-E. 2003. Stochastic Population Dynamics in Ecology and Conservation. Oxford (United Kingdom): Oxford University Press.

Latimer A. M., Silander J. A. Jr., Cowling R. M. 2005. Neutral ecological theory reveals isolation and rapid speciation in a biodiversity hot spot. Science 309:1722-1725.

Lavin M., Schrire B. P., Lewis G., Pennington R. T., Delgado-Salinas A., Thulin M., Hughes C. E., Beyra Matos A., Wojciechowski M. F. 2004. Metacommunity process rather than continental tectonic history better explains geographically structured phylogenies in legumes. Philosophical Transactions of the Royal Society B: Biological Sciences 359:1509-1522.

Leibold M. A. 2008. Return of the niche. Nature 454: 39-40.

Leibold M. A., McPeek M. A. 2006. Coexistence of the niche and neutral perspectives in community ecology. Ecology 87:1399-1410.

Leigh E. G. Jr. 2007. Neutral theory: a historical perspective. Journal of Evolutionary Biology 20:20752091.

Lessard J.-P., Belmaker J., Myers J. A., Chase J. M., Rahbek C. 2012. Inferring local ecological processes amid species pool influences. Trends in Ecology and Evolution 27:600-607.

Levine J. M., HilleRisLambers J. 2009. The importance of niches for the maintenance of species diversity. Nature 461:254-257.

Levins R. 1966. The strategy of model building in population biology. American Scientist 54:421-431.

Lewis D. 1973. Causation. Journal of Philosophy 70: 556-567.

Lortie C. J., Brooker R. W., Choler P., Kikvidze Z., Michalet R., Pugnaire F. I., Callaway R. M. 2004. Rethinking plant community theory. Oikos 107 : 433-438.

Louda S. M., Keeler K. H., Holt R. D. 1990. Herbivore influences on plant performance and competitive interactions. Pages 413-444 in Perspectives on Plant Competition, edited by J. B. Grace and D. Tilman. San Diego (California): Academic Press.

MacArthur R., Levins R. 1967. The limiting similarity, convergence, and divergence of coexisting species. American Naturalist 101:377-385.

McGill B. 2003. Strong and weak tests of macroecological theory. Oikos 102:679-685.

McGill B. J., Nekola J. C. 2010. Mechanisms in macroecology: AWOL or purloined letter? Towards a pragmatic view of mechanism. Oikos 119:591-603.

McGill B. J., Maurer B. A., Weiser M. D. 2006a. Empirical evaluation of neutral theory. Ecology 87: 1411-1423.

McGill B. J., Enquist B. J., Weiher E., Westoby M. 2006b. Rebuilding community ecology from functional traits. Trends in Ecology and Evolution 21:178-185. 
Menzies P. 2004. Causal models, token causation, and processes. Philosophy of Science 71:820-832.

Mikkelson G. M. 2005. Niche-based vs. neutral models of ecological communities. Biology and Philosophy 20:557-566.

Mouquet N., Loreau M. 2003. Community patterns in source-sink metacommunities. American Naturalist 162:544-557.

Mouquet N., Devictor V., Meynard C. N., et al. 2012. Ecophylogenetics: advances and perspectives. Biological Reviews 87:769-785.

Mouquet N., Lagadeuc Y., Devictor V., et al. 2015. Predictive ecology in a changing world. Journal of Applied Ecology 52:1293-1310.

Muneepeerakul R., Bertuzzo E., Lynch H. J., Fagan W. F., Rinaldo A., Rodriguez-Iturbe I. 2008. Neutral metacommunity models predict fish diversity patterns in Mississippi-Missouri basin. Nature 453: 220-222.

Munoz F., Ramesh B. R., Couteron P. 2014. How do habitat filtering and niche conservatism affect community composition at different taxonomic resolutions? Ecology 95:2179-2191.

Noble A. E., Temme N. M., Fagan W. F., Keitt T. H. 2011. A sampling theory for asymmetric communities. Journal of Theoretical Biology 273:1-14.

Ohta T. 1992. The nearly neutral theory of molecular evolution. Annual Review of Ecology and Systematics 23:263-286.

Preston F. W. 1948. The commonness, and rarity, of species. Ecology 29:254-283.

Pueyo S. 2006. Diversity: between neutrality and structure. Oikos 112:392-405.

Purves D. W., Pacala S. W. 2005. Ecological drift in niche-structured communities: neutral pattern does not imply neutral process. Pages 107-140 in Biotic Interactions in the Tropics: Their Role in the Maintenance of Species Diversity, edited by D. F. R. P. Burslem, M. A. Pinard, and S. E. Hartley. Cambridge (United Kingdom): Cambridge University Press.

Purves D. W., Turnbull L. A. 2010. Different but equal: the implausible assumption at the heart of neutral theory. Journal of Animal Ecology 79:1215-1225.

Ricklefs R. E. 2008. Disintegration of the ecological community. American Naturalist 172:741-750.

Rosindell J., Cornell S. J., Hubbell S. P., Etienne R. S. 2010. Protracted speciation revitalizes the neutral theory of biodiversity. Ecology Letters 13:716727.

Rosindell J., Hubbell S. P., Etienne R. S. 2011. The unified neutral theory of biodiversity and biogeography at age ten. Trends in Ecology and Evolution 26:340-348.

Salmon W. C. 1984. Scientific Explanation and the Causal Structure of the World. Princeton (New Jersey): Princeton University Press.
Scheffer M., van Nes E. H. 2006. Self-organized similarity, the evolutionary emergence of groups of similar species. Proceedings of the National Academy of Sciences of the United States of America 103:62306235.

Shipley B., Vile D., Garnier É. 2006. From plant traits to plant communities: a statistical mechanistic approach to biodiversity. Science 314:812-814.

Sober E. 1981. The principle of parsimony. British Journal for the Philosophy of Science 32:145-156.

Sober E. 1993. The Nature of Selection: Evolutionary Theory in Philosophical Focus. Cambridge (Massachusetts): MIT Press.

Strevens M. 2004. The causal and unification approaches to explanation unified-causally. Noûs 38 : 154-176.

Tilman D. 1994. Competition and biodiversity in spatially structured habitats. Ecology 75:2-16.

Ulrich W., Gotelli N. J. 2013. Pattern detection in null model analysis. Oikos 122:2-18.

Vellend M. 2010. Conceptual synthesis in community ecology. Quarterly Review of Biology 85:183-206.

Vergnon R., Dulvy N. K., Freckleton R. P. 2009. Niches versus neutrality: uncovering the drivers of diversity in a species-rich community. Ecology Letters 12:1079-1090.

Veuille M. 2000. Genetics and the evolutionary process. Comptes Rendus de l'Académie des Sciences-Series III-Sciences de la Vie 323:1155-1165.

Volkov I., Banavar J. R., Hubbell S. P., Maritan A. 2003. Neutral theory and relative species abundance in ecology. Nature 424:1035-1037.

Walker S. C. 2007. When and why do non-neutral metacommunities appear neutral? Theoretical Population Biology 71:318-331.

Waters C. K. 2007. Causes that make a difference. Journal of Philosophy 104:551-579.

Wennekes P. L., Rosindell J., Etienne R. S. 2012. The neutral-niche debate: a philosophical perspective. Acta Biotheoretica 60:257-271.

Whitfield J. 2002. Neutrality versus the niche. Nature 417:480-481.

Wilson J. B. 1999. Guilds, functional types and ecological groups. Oikos 86:507-522.

Woodward J. 2003. Experimentation, causal inference, and instrumental realism. Pages 87-118 in The Philosophy of Scientific Experimentation, edited by H. Radder. Pittsburgh (Pennsylvania): University of Pittsburgh Press.

Zhou S.-R., Zhang D.-Y. 2008. A nearly neutral model of biodiversity. Ecology 89:248-258.

Associate Editor: Mark Dale Handling Editor: Daniel E. Dykhuizen 